\title{
PRIMER REGISTRO DEL GÉNERO ZALOPHORA HUNT, 1994 (NEMATODA: XUSTROSTOMATIDAE) PARA CUBA Y DESCRIPCIÓN DE UNA NUEVA ESPECIE
}

\author{
Nayla García Rodríguez y Jans Morffe Rodríguez \\ Instituto de Ecología y Sistemática. Carretera de Varona, km 31⁄2, Capdevila, Boyeros, Ciudad de La \\ Habana, Cuba.nayla@ecologia.cu. jans@ecologia.cu.
}

\section{RESUMEN}

Se describe una nueva especie de Zalophora Hunt, 1994 (Nematoda: Xustrostomatidae) parásita de milpiés (Diplopoda: Spirobolida) en La Gran Piedra, provincia Santiago de Cuba, Cuba. Se describen los machos del género por primera vez. Este constituye el primer registro de este género y familia para Cuba y las Antillas.

Palabras clave: Nematoda, Diplopoda, Zalophora, nueva especie, Antillas, Cuba.

\section{ABSTRACT}

A new species belonging to genus Zalophora Hunt, 1994 (Nematoda: Xustrostomatidae) is described from a millipede (Diplopoda: Spirobolida) from La Gran Piedra, Santiago de Cuba province, Cuba. Males of the genus are described for the first time. This is the first record of the genus and the family for Cuba and the Antilles.

Key words: Nematoda, Diplopoda, Zalophora, new species, West Indies, Cuba.

\section{INTRODUCCIÓN}

Hunt (1994) estableció el género Zalophora para un rigonemátido (Rhigonematida: Rhigonematidae), parásito de un pequeño milpiés (Diplopoda: Spirobolida) de Papua, Nueva Guinea. Este género se caracteriza por la peculiar estructura de la región cefálica de las hembras, rematada por crestas dorsoventrales sobre la apertura oral e incluye tres especies: Z. hepialum Hunt, 1994 (especie tipo), Z. deinostoma Hunt et Moore, 1999 y Z. nemophila Hunt et Moore, 1999 (Hunt, 1994; Hunt y Moore, 1999). En el arreglo sistemático de la familia Rhigonematidae este género fue reubicado por su autor en Xustrostomatidae, junto a Obainia Adamson, 1983, Trachyglossus Hunt, 2002 y Xustrostoma Adamson et Waerebeke, 1984 (Hunt, 2002).

En el presente trabajo se describe una nueva especie de Zalophora, lo que también constituye el primer registro del género y la familia para Cuba y las Antillas; además, se ofrece la descripción de los machos, desconocidos hasta el momento en las especies de este género.

\section{MATERIALES Y MÉTODOS}

Se recolectaron de forma manual en hojarasca y troncos en descomposición, cinco ejemplares de Nesobolus piedra Pérez-Asso, 1996 (Diplopoda: Spirobolida: Rhinocricidae) procedentes de los alrededores del cafetal La Isabelica, La Gran Piedra, provincia Santiago de Cuba, Cuba, en abril del 2009. Los hospederos se llevaron vivos al laboratorio en frascos con hojarasca del sitio de colecta y después se sacrificaron por inmersión en etanol al $70 \%$ o con vapores de éter etílico. Los intestinos se extrajeron inmediatamente mediante un corte en los últimos segmentos del cuerpo. Se disecaron en solución salina fisiológica para extraer los parásitos, que se sacrificaron con agua caliente $\left(60-80^{\circ} \mathrm{C}\right)$ y se fijaron en etanol al $70 \%$.

Para su examen, los nemátodos se montaron en portaobjetos con glicerina anhidra y se sellaron los bordes del cubreobjetos con esmalte de uñas. La observación se llevó a cabo en un microscopio óptico con aumentos de 40x a 400x y las mediciones, con un micrómetro ocular de escala lineal, con 
una precisión de $0,001 \mathrm{~mm}$. Las variables morfométricas tomadas fueron las utilizadas por Hunt (2002). De cada medida se ofrece su rango seguido por la media y la desviación estándar, entre paréntesis. Todos los valores están expresados en milímetros. Se calcularon los índices de De Man a, b, c y V\% (De Man, 1884).

Los ejemplares se fotografiaron con una cámara digital AxioCam acoplada a un microscopio Carl Zeiss AxioScop 2 Plus. Los dibujos se realizaron en CorelDRAW X3 y Adobe Photoshop CS2 sobre la base de las microfotografías. Las escalas de las ilustraciones están dadas en milímetros.

El material estudiado se encuentra depositado en la Colección Helmintológica de las Colecciones Zoológicas del Instituto de Ecología y Sistemática (CZACC), La Habana, Cuba.

\author{
RESULTADOS \\ SISTEMÁTICA \\ Xustrostomatidae \\ Zalophora Hunt, 1994 \\ Zalophora repentina sp. nov. \\ (Fig.1 A-K, Fig. 2 A-F)
}

Diagnosis. Hembras robustas, de pequeño tamaño. Extremo cefálico característico del género, con una constricción hacia el final del esófago. Crestas bien desarrolladas, no lobuladas. Vulva algo desplazada hacia la mitad posterior del cuerpo. Cola con un ensanchamiento redondeado en la región media, entre la base y el extremo apical. Machos más pequeños que las hembras, con la región cefálica similar a estas. Espículas desiguales en forma; la derecha curva, con una constricción cerca de la base. Nueve pares de papilas precloacales y tres pares de papilas postcloacales.

Diagnosis. Females with body stout and small. Cephalic end characteristic of genus, with a constriction in the end of esophagus. Crests well developed and not lobated. Vulva displaced to the posterior half of body. Tail with a rounded expansion in the middle between anus and the end tip. Males smaller than females, with the cephalic end similar to these. Spicules unequal in shape. Right spicule more curved, with a constriction near the base. Nine pairs of pre-cloacal papillae and three pairs of post-cloacal papillae present.

Descripción. Hembras. Nemátodos de pequeño tamaño y apariencia robusta. Cutícula muy finamente estriada hasta la cola. Extremo cefálico globular y ensanchado. Apertura bucal triangular, rodeada por dos crestas cefálicas en posición dorsoventral y poco lobuladas. Anfidios con las aberturas dorsolaterales en los extremos de las crestas. Cuerpo con una constricción hacia el final del esófago. Cuerpo esofágico compacto, marcadamente musculoso, ancho, subglobular, amalgamado al bulbo, también musculoso, ensanchado y de menor diámetro que el cuerpo esofágico. En el comienzo del cuerpo esofágico presenta estructuras cuticulares esclerosadas, semejantes a mandíbulas, provistas de hileras de dientes pequeños y puntiagudos. Intestino simple, subrectilíneo, con la porción anterior no dilatada. Recto corto y ano no sobresaliente. Sistema reproductor didelfo, ovarios opuestos y reflexos. Vulva con los labios no sobresalientes, algo desplazada hacia la mitad posterior del cuerpo, dirigida hacia el extremo anterior. Huevos numerosos, casi esféricos, con la cáscara fina y lisa. Cola cónica, corta, atenuada y con un ensanchamiento redondeado en la región media entre la base y el extremo apical, terminado en punta.

Machos. Más pequeños que las hembras, con la región cefálica similar a estas. Esófago similar a las hembras pero menos robusto. Testis reflexo. Espículas desiguales, pequeñas, cortas, relativamente estrechas. Espícula izquierda casi recta y más pequeña. Espícula derecha algo curva, con una constricción cerca de la base. Nueve pares de papilas precloacales subventrales; los ocho primeros pares son equidistantes y el noveno par está separado del octavo por casi el doble de la distancia entre los pares anteriores. Tres pares de papilas postcloacales pequeñas; los dos primeros sublaterales y próximos entre sí y el último subventral y situado en la base de la cola. Cola corta, cónica y algo curvada en posición ventral. 

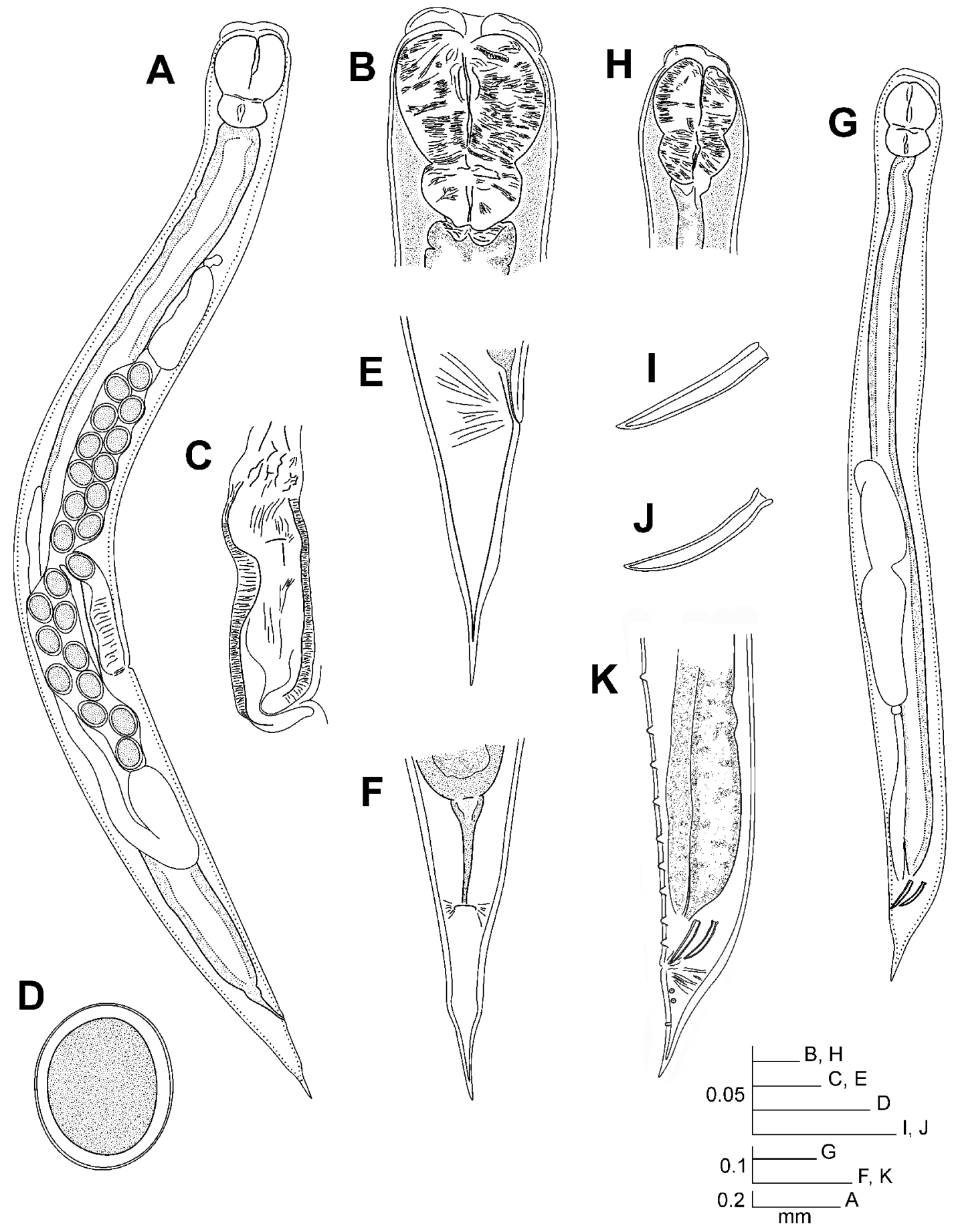

Figura 1. Zalophora repentina sp. nov. (dibujos). A. Habitus de la hembra. B. Región cervical de la hembra, vista ventral. C. Ovoyector. D. Huevo. E. Cola de la hembra, vista lateral. F. Cola de la hembra, vista ventral. G. Habitus del macho. H. Región cervical del macho, vista lateral. I. Espícula izquierda. J. Espícula derecha. K. Cola del macho, vista lateral. 
Tipos. Holotipo (hembra) CZACC 11.4504, en Nesobolus piedra; Isabelica, La Gran Piedra, Santiago de Cuba, Cuba; abril de 2009; J. Morffe col. Alotipo (macho) CZACC 11.4505, idénticos datos que el holotipo. Paratipo (hembra) CZACC 11.4506, idénticos datos que el holotipo.

Medidas. Holotipo (hembra) $\mathrm{a}=12.88 ; \mathrm{b}=13.21 ; \mathrm{c}=12.88 ; \mathrm{V} \%=61.17$; longitud total $=2.575$; ancho máximo $=0.200$; longitud del cuerpo esofágico $=0.135$; diámetro del bulbo esofágico $=0.110$; longitud total del esófago $=0.195$; distancia de la vulva al extremo de la cola $=1.100$; distancia del ano al extremo de la cola $=0.200$; huevos $(\mathrm{n}=5)$ 0.073-0.080 $\times 0.055-0.063(0.077 \pm 0.003 \times 0.059 \pm 0.003)$. Alotipo (macho) $\mathrm{a}=11.54 ; \mathrm{b}=12.00 ; \mathrm{c}=12.50 ;$ longitud total $=1.500 ;$ ancho máximo $=0.130 ;$ longitud del cuerpo esofágico $=0.075$; diámetro del bulbo esofágico $=0.078$; longitud total del esófago $=0.125$; distancia del ano al extremo de la cola $=0.120$; longitud de la espícula izquierda $=0.063 ;$ longitud de la espícula derecha $=0.060$. Paratipo (hembra) $\mathrm{a}=12.50 ; \mathrm{b}=15.33 ; \mathrm{c}=14.38$; longitud total $=2.875$; ancho máximo $=0.230$; longitud del cuerpo esofágico $=0.125$; diámetro del bulbo esofágico $=0.110$; longitud total del esófago $=$ 0.188 ; distancia del ano al extremo de la cola $=0.200$; huevos $(\mathrm{n}=5) 0.063-0.085 \times 0.058-0.063(0.075 \pm$ $0.010 \times 0.061 \pm 0.002)$.
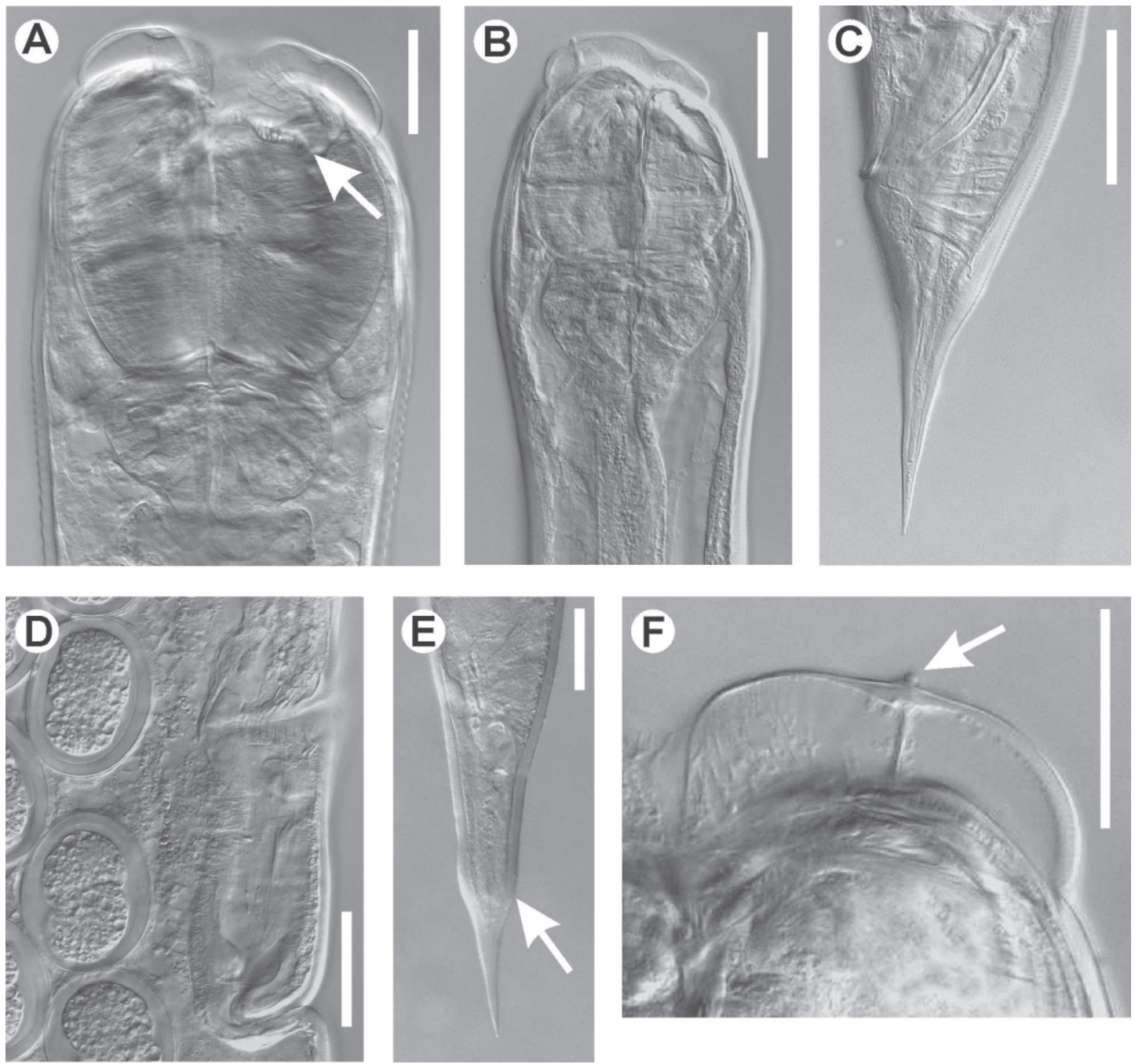

Figura 2. Zalophora repentina sp. nov. (fotografías) A. Región cervical de la hembra (la flecha señala las estructuras similares a mandíbulas al comienzo del cuerpo esofágico). B. Región cervical del macho. C. Cola y espícula derecha del macho. D. Ovoyector y huevos. E. Cola de la hembra, vista ventral (la flecha señala el ensanchamiento entre el ano y el extremo de la cola). F. Apertura del anfidio en el extremo de la cresta cefálica. Líneas de escala $0.05 \mathrm{~mm}$. 
Hospedante tipo. Nesobolus piedra Pérez-Asso, 1996 (Diplopoda: Spirobolida). Localización: intestino posterior.

Etimología. Epíteto específico derivado del latín repentina, sorpresa. Referido a lo sorprendente del hallazgo en esta área geográfica del género Zalophora, conocido con anterioridad sólo para Nueva Guinea.

\section{DISCUSIÓN}

Zalophora repentina $\mathrm{sp}$. nov. es la más pequeña de las especies del género, sólo alcanza la mitad de la longitud total $(2.725 \mathrm{~mm})$ de la especie de menor talla, Z. deinostoma $(4.335 \mathrm{~mm})$. La cola difiere de las otras especies en la forma, por el característico ensanchamiento en su región media. Por otra parte la vulva está ubicada más posteriormente que en el resto. De Z. nemophila se diferencia, además, por tener la cola proporcionalmente más larga $(\mathrm{c}=12.88-14.38 ; 31.1)$. De $Z$. hepialum por tener el esófago más largo con relación a la longitud corporal $(\mathrm{b}=13.21-15.33 ; 17.6$ 19.3) y por tener el margen de las crestas no lobulado. De Z. deinostoma por tener las crestas mucho más desarrolladas.

Los machos de Zalophora repentina sp. nov. son los primeros descritos para el género y presentan espículas muy diferentes en cuanto a su forma, carácter que difiere del establecido por Hunt (2002) para la familia. La presencia de Zalophora repentina sp. nov. constituye el primer registro de rigonemátido para el género Nesobolus Chamberlin, 1918.

\section{AGRADECIMIENTOS}

A la Lic. Tamara Tcherva por la identificación de los hospedantes. Al MSc. Yamir Torres y MSc. Eduardo Furrazola, del Instituto de Ecología y Sistemática por la realización de las microfotografías. A IDEAWILD por su donativo para el trabajo de campo y laboratorio. Este resultado está inscrito en el proyecto Colecciones Zoológicas, su conservación y manejo, del Programa Ramal de Ciencia y Técnica de Diversidad Biológica del Ministerio de Ciencia, Tecnología y Medio Ambiente.

\section{LITERATURA CITADA}

De Man, J.G. 1884. Die frei der reinen Erde und in sussen Wasser Lebenden nematoden neiderlandischen fauna, Eine Systematische Faunistische Monographie. Leiden, 206 pp.

Hunt, D. J. 1994. Zalophora hepialum, a new genus and species of rhigonematid (Nematoda, Rhigonematidae) in diplopod from Papua New Guinea. Acta Parasitologica, 39(4):197-200.

Hunt, D. J. 2002. The African Rhigonematoidea (Nematoda:Rhigonematida).1. Trachyglossus quintus gen.n.,sp. N. (Xustrostomatidae fam.n.) with redescription of Rhigonema multipapillatum (Skrjabin,1916) and designation of a neotype. Nematology, 4(6):671-686.

Hunt, D.J. y D. Moore. 1999. Rhigonematida from New Britain diplopods.2. The genera Rhigonema Cobb,1898 and Zalophora Hunt,1994 (Rhigonematoidea: Rhigonematidae) with descriptions of three new species. Nematology, 1(13):225-242. 